\title{
Segmentation of sales for a mobile phone service through CART classification tree algorithm
}

\author{
Autores \\ Amelec Viloria, Guojun Wang, Mercedes Gaitan
}

\begin{abstract}
The work consisted of detailing the CRISP-DM method in order to identify optimal groups of customers who are more likely to migrate from a prepaid to postpaid option in order to formulate an improvement plan for in call management by sorting the database. Classification models were applied to analyze the characteristics generated by the purchase of the different services. The CART Classification Tree algorithm. As a result, groups differentiated by probabilities of sales success (migrate from a prepaid to postpaid plan) were found, segments that reflect particular needs and characteristics to design marketing actions focused on the objective of increasing the effectiveness rate, contact information, and sales increase.
\end{abstract}

Palabras clave

Call center, CRISP-DM CART, classification tree algorithm 